\title{
The Spatial Sensitivity Analysis of Evapotranspiration using Penman-Monteith Method at Grid Scale
}

\author{
Sivarajah Mylevaganam*, Chittaranjan Ray \\ University of Nebraska-Lincoln, Lincoln, NE, USA \\ Email: 'sivaloga@hushmail.com
}

Received 26 December 2016; accepted 26 February 2016; published 29 February 2016

Copyright (C) 2016 by authors and Scientific Research Publishing Inc.

This work is licensed under the Creative Commons Attribution International License (CC BY). http://creativecommons.org/licenses/by/4.0/

c) (i) Open Access

\section{Abstract}

The need to allocate the existing water in a sustainable manner, even with the projected population growth, has made to assess the consumptive use or evapotranspiration (ET), which determines the irrigation demand. As underscored in the literature, Penman-Monteith method which is a combination of aerodynamic and energy balance method is widely used and accepted as the method of estimation of ET. However, the application of Penman-Monteith relies on many climate parameters such as relative humidity, solar radiation, temperature, and wind speed. Therefore, there exists a need to determine the parameters that are most sensitive and correlated with dependent variable (i.e., ET), to strengthen the knowledge base. However, the sensitivity of ET using Penman-Monteith is oftentimes estimated using meteorological data from climate stations. Such estimation of sensitivity may vary spatially and thus there exists a need to estimate sensitivity of ET spatially. Thus, in this paper, based on One-AT-A-Time (OAT) method, a spatial sensitivity tool that can geographically encompass all the best available climate datasets to produce ET and its sensitivity at different spatial scales is developed. The spatial tool is developed as a Python toolbox in ArcGIS using Python, an open source programming language, and the ArcPy site-package of ArcGIS. The developed spatial tool is demonstrated using the meteorological data from Automated Weather Data Network in Nebraska in 2010. To summarize the outcome of the sensitivity analysis using OAT method, sensitivity indices are developed for each raster cell. The demonstration of the tool shows that, among the considered parameters, the computed ET using Penman-Monteith is highly sensitive to solar radiation followed by temperature for the state of Nebraska, as depicted by the sensitivity index. The computed sensitivity index of wind speed and the relative humidity are not that significant compared to the sensitivity index of solar radiation and temperature.

\section{Keywords}

Evapotranspiration, Penman-Monteith Method, Aerodynamic Method, Energy Balance Method,

${ }^{*}$ Corresponding author.

How to cite this paper: Mylevaganam, S. and Ray, C. (2016) The Spatial Sensitivity Analysis of Evapotranspiration using Penman-Monteith Method at Grid Scale. Journal of Geographic Information System, 8, 121-136. 
Python, ArcPy, ArcGIS, Spatial Scale, Geoprocessing, Python Toolbox, Sensitivity Analysis, One-AT-A-Time, Sensitivity Index

\section{Introduction}

Although over 70\% of Earth's surface is covered by water, the amount of freshwater available for appropriation is limited as $97.5 \%$ of all water on Earth is saline [1]. This limited freshwater is competed by many water users such as irrigation, household and municipal, and industrial. Among these water users, irrigation demand, which is a function of evapotranspiration (ET), the rate of liquid water transformation to vapor from open water, bare soil, and vegetation, accounts for $87 \%$ of the total use globally [2]. However, in the next few years with the projected population growth, even under the assumption that the consumption of water per capita remains constant, the water demands increase and thus multitude the water allocation problems many fold [2] [3]. Therefore, to allocate the existing water in a sustainable manner, there is a need to estimate ET.

As underscored in the literature [4]-[13], to date, there are many methods available to estimate ET. These methods are either empirical or climate data driven. Under empirical based estimation of ET, Blaney-Criddle method or its modified version is widely used in the arid western regions of the United States [4] [5]. However, this method doesn't account for humidity, wind speed, and other climate factors. On the other hand, using meteorological data from climate stations, the methods of estimation of ET include aerodynamic method, energy balance method, and combination methods such as Penman-Monteith method [4]-[6]. The aerodynamic method of determining evaporation considers the transport of water vapor by the turbulence of the wind blowing over a natural surface. The energy balance method considers all heat energy received and reflected/dissipated by a cropped area or a water body. Penman-Monteith method of evaporation is obtained by combining the evaporation computed by aerodynamic and energy balance method [4]-[6]. As underscored in the literature [4]-[13], Penman-Monteith method is widely used and accepted as the method of estimation of ET. However, the application of Penman-Monteith relies on many climate parameters such as relative humidity, solar radiation, temperature, and wind speed. Therefore, there exists a need to determine the parameters that are most influential and correlated with dependent variable (i.e., ET), to strengthen the knowledge base [14]. Oftentimes, sensitivity analysis is conducted to identify these parameter sets.

Based on a 20-yr historical daily climate dataset, in computing ET using FAO56 Penman-Monteith method [6], [7] shows that short wave radiation was the most influential climate variable for Ejina Oasis (North West China), followed by air temperature, wind speed, and relative humidity. [8] found that the response of ET in Changjiang (Yangtze river basin, China) was precisely predicted under perturbation of relative humidity or short wave radiation by their sensitivity coefficients. This study also concludes that wind speed had the least impact on ET. Using the data from the automatic meteorological station of Aminteo in the Prefecture of Florina, Western Macedonia, Greece, [9] shows that solar radiation and temperature are the main climate parameters that affect ET, while relative humidity and wind speed are not so important for the calculation of ET. With global multivariate technique over the whole Toce basin (Italian Alps), [10] concludes that aerodynamic parameters were less critical in predicting ET. A study with 22 years of meteorological data at station Fasa, Iran, shows that the estimated ET is sensitive to net radiation, maximum temperature, and wind speed [11]. The research conducted by [12] in an experimental farm of the Shahid Bahonar, University of Kerman, Iran, shows that ET is sensitive to vapor pressure deficit followed by wind speed, but during the summer ET is sensitive to short wave radiation. Zha et al. (2013) found that ET over a Scots pine forest in eastern Finland, is sensitive to radiation, vapor pressure deficit, and temperature. This study also concludes that though the radiation explained $42 \%$ of variation in ET, it cannot alone accurately predict the estimation of ET.

As presented in the previous paragraph, the sensitivity of ET using Penman-Monteith is oftentimes estimated using meteorological data from climate stations. However, such estimation of sensitivity may vary spatially and thus there exists a need to estimate sensitivity of ET spatially. Thus, the objectives of this paper are to a) develop a spatial sensitivity tool that can geographically encompass all the best available climate datasets using Python, an open source programming language supported by a growing user community for its extensive collection of standard and third-party libraries, and the ArcPy site-package of ArcGIS, b) evaluate the spatial sensitivity of ET using Penman-Monteith c) evaluate the spatial sensitivity of ET using aerodynamic method and energy balance method. 


\section{Estimation of Evapotranspiration}

The need to manage the available freshwater wisely with ever increasing population and the demand from irrigation has brought ET as one of the critical subject areas to research in the field of hydrology. Over the years, with many research works, numerous methods have been developed to estimate ET. These methods mainly fall under these categories: a) aerodynamic method, b) energy balance method, and c) combination of aerodynamic and energy balance methods [4]-[13].

\subsection{Aerodynamic Method}

This method of determining evaporation considers the transport of water vapor by the turbulence of the wind blowing over a natural surface. According to this method, the evaporation $\left(E_{a}\right)$, generally from lakes and reservoirs, is proportional to $\left(e_{s}-e_{z}\right)$. The mathematical expression of this method is given by Equation (1).

$$
E_{a}=M *\left(e_{\mathrm{s}}-e_{\mathrm{z}}\right) * u_{\mathrm{z}}
$$

where $M, e_{s}, e_{z}$ and $u_{z}$ are mass transfer coefficient, saturated vapor pressure at water temperature, vapor pressure at height $z$, and wind velocity at height $z$, respectively. The mass transfer coefficient is given by Equation (2).

$$
M=0.622 * \frac{\rho_{a} C_{E}}{\rho_{w} P}
$$

where $P, \rho_{w}, \rho_{a}$ and $C_{E}$ are atmospheric pressure at height $z$, density of water, density of air, and evaporation coefficient, respectively.

By substituting Equation (2) in Equation (1),

$$
E_{a}=0.622 * \frac{\rho_{a} C_{E}}{\rho_{w} P} *\left(e_{s}-e_{z}\right) * u_{z}
$$

Considering aerodynamic resistance, $r_{a}=\frac{1}{C_{E} u_{z}}$, Equation (3) leads to

$$
E_{a}=0.622 * \frac{\rho_{a}}{\rho_{w} P r_{a}} *\left(e_{s}-e_{z}\right)
$$

Since $\rho_{a}=\frac{3.486 P}{T+273}$, Equation (4) becomes

$$
E_{a}=0.622 * \frac{3.486 P}{\rho_{w} P r_{a} *(T+273)} *\left(e_{s}-e_{z}\right)
$$

where $T$ is the air temperature in degree Celsius. With further simplification,

$$
E_{a}=\frac{2.17\left(e_{s}-e_{z}\right)}{\rho_{w} r_{a} *(T+273)}
$$

\subsection{Energy Balance Method}

As shown in Figure 1, this method considers all heat energy received and reflected/dissipated by a cropped area or a water body. The portion of energy that is used to warm the air in contact with the ground or water surface is known as sensible heat flux $(H)$. The term $G$ is the heat conduction from the water surface or soil to the layer of soil or water below. Since the energy required to evaporate a unit mass of water is called latent heat of vaporization $(\lambda)$, the total energy absorbed per unit area to evaporate $E_{r}$ is $\rho_{w} \lambda E_{r}$. Therefore, neglecting the other small energy terms that are dissipated/stored, the energy balance for the control volume shown in Figure 1 is given by Equation (6).

$$
R_{n}-H-G-\rho_{w} \lambda E_{r}=0
$$




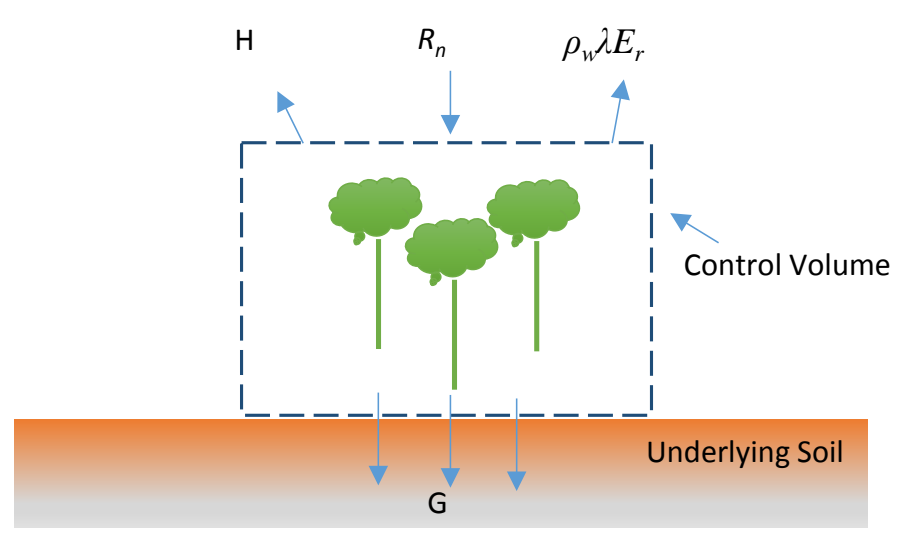

\section{Figure 1. The energy flow diagram for a cropped area.}

where $R_{n}$ is the net radiation. The latent heat of vaporization is $2.45 \mathrm{MJ} / \mathrm{kg}$ at about 20 degree Celsius [6]. However, to account for temperature variation, the latent heat of vaporization is given by

$$
\lambda=2.501-0.002362 T
$$

The sensible heat flux defined by Equation (7) is related to Bowen ratio, $\beta$

$$
H=\beta * \rho_{w} \lambda E_{r}
$$

Bowen ratio, $\beta=\gamma \frac{T_{2}-T_{1}}{e_{2}-e_{1}}$ is derived from temperatures and vapor pressures at two heights above the water surface. By substituting Equation (7) in Equation (6),

$$
E_{r}=\frac{R_{n}-G}{\rho_{w} \lambda(1+\beta)}
$$

Since $G \approx 0$,

$$
E_{r}=\frac{R_{n}}{\rho_{w} \lambda(1+\beta)}
$$

As shown in Equation (10), the net radiation $\left(R_{n}\right)$ is the sum of net long-wave radiation $\left(L_{n}\right)$ and net short-wave radiation $\left(S_{n}\right)$.

$$
R_{n}=L_{n}+S_{n}
$$

The net short-wave radiation that is defined by Equation (11) is a function of total extraterrestrial radiation $\left(S_{0}\right)$ and cloudiness fraction $\left(\frac{n}{N}\right)$.

$$
S_{n}=(1-\alpha)\left(0.25+0.5 \frac{n}{N}\right) S_{0}
$$

The net long-wave radiation which is in accord with Stefan-Boltzmann's law of black body radiation is given by Equation (12).

$$
L_{n}=-\left(0.1+0.9 \frac{n}{N}\right)\left(0.34-0.14 \sqrt{e_{d}}\right) \sigma T^{4}
$$

where $e_{d}, \sigma$ and $T$ are vapor pressure at air temperature, Stefan-Boltzmann constant, and mean air temperature, respectively.

\subsection{Combination Method of Penman}

As shown in Equation (13), this method of evaporation is obtained by combining the evaporation computed by 
aerodynamic ( $E_{a}$ ) and energy balance method $\left(E_{r}\right.$ ). The weighting factors (i.e., $\frac{\Delta}{\Delta+\gamma}$ and $\frac{\gamma}{\Delta+\gamma}$ ) are applied in combining the methods (i.e., aerodynamic and energy balance). The weighting factors sum to unity.

$$
E=\frac{\Delta}{\Delta+\gamma} E_{r}+\frac{\gamma}{\Delta+\gamma} E_{a}
$$

where $\gamma$ is the psychrometric constant that is defined by Equation (14). The gradient of the saturated vapor pressure $(\Delta)$ is given by Equation (15).

$$
\begin{aligned}
& \gamma=0.0016286 \frac{P}{\lambda} \\
& \Delta=\frac{4098 e_{s}}{(273.3+T)^{2}}
\end{aligned}
$$

\subsection{Penman-Monteith Method}

This method is same as the combination method of Penman. However, in this method, similar to $r_{a}$, another term called surface resistance $\left(r_{s}\right)$ is introduced to account for resistance associated with movement of water vapor from the plant leaves to the air outside. This method is widely used to estimate evapotranspiration. The mathematical expression of this method is given by Equation (16).

$$
E=\frac{\Delta}{\Delta+\gamma *\left(1+\frac{r_{s}}{r_{a}}\right)} * \frac{R_{n}-G}{\rho_{w} \lambda}+\frac{\gamma}{\Delta+\gamma *\left(1+\frac{r_{s}}{r_{a}}\right)} * \frac{2.17\left(e_{s}-e_{z}\right)}{\rho_{w} r_{a} *(T+273)}
$$

For grass reference crop, $r_{\mathrm{s}}=69 \mathrm{~s} / \mathrm{m}$ and $r_{a}=\frac{208}{u_{2}} \mathrm{~s} / \mathrm{m}$. Therefore, for grass reference crop $1+\frac{r_{s}}{r_{a}}=1+0.33 u_{2}$.

where $u_{2}$ is the wind speed at $2 \mathrm{~m}$. When the wind speed is measured at different elevation, it can be adjusted from one level to another by using Equation (17).

$$
u_{2}=u_{1} * \frac{\ln \frac{z_{2}}{z_{0}}}{\ln \frac{z_{1}}{z_{0}}}
$$

where $Z_{1}, Z_{2}$ are measurement heights for levels 1 and 2 , respectively. $Z_{0}$ is the reference height where velocity is zero. For open agricultural area, $Z_{0}=0.03$. The inner details of the method are presented in Figure 2.

\section{Sensitivity Analysis}

The mathematical model of a dependent variable $\left(Y=f\left(X_{1}, X_{2}, \cdots, X_{n}\right)\right)$ is formed by means of model parameters/independent variables $\left(X_{1}, X_{2}, \cdots, X_{n}\right)$ that define the underlying processes. Therefore, there exists a need to determine the parameters that are most influential on model outcome and mostly correlated with model output. Oftentimes, sensitivity analysis is conducted to identify the influential parameter sets. In general, sensitivity analysis methods are pooled under two categories, namely local and global methods. The local sensitivity analysis is the simplest though the combined variability resulting from changing all the parameters simultaneously is precluded. Under the local sensitivity analysis method, there are many methods to gauge the sensitivity analysis depending on the purpose. The differential sensitivity analysis and the One-AT-A-Time (OAT) method are the most widely used local sensitivity methods. The differential sensitivity analysis is based on partial differential equations. This method is efficient if an explicit relationship exists between dependent and independent variables. However, the computational effort is intensive and the implementation is complex if the function 


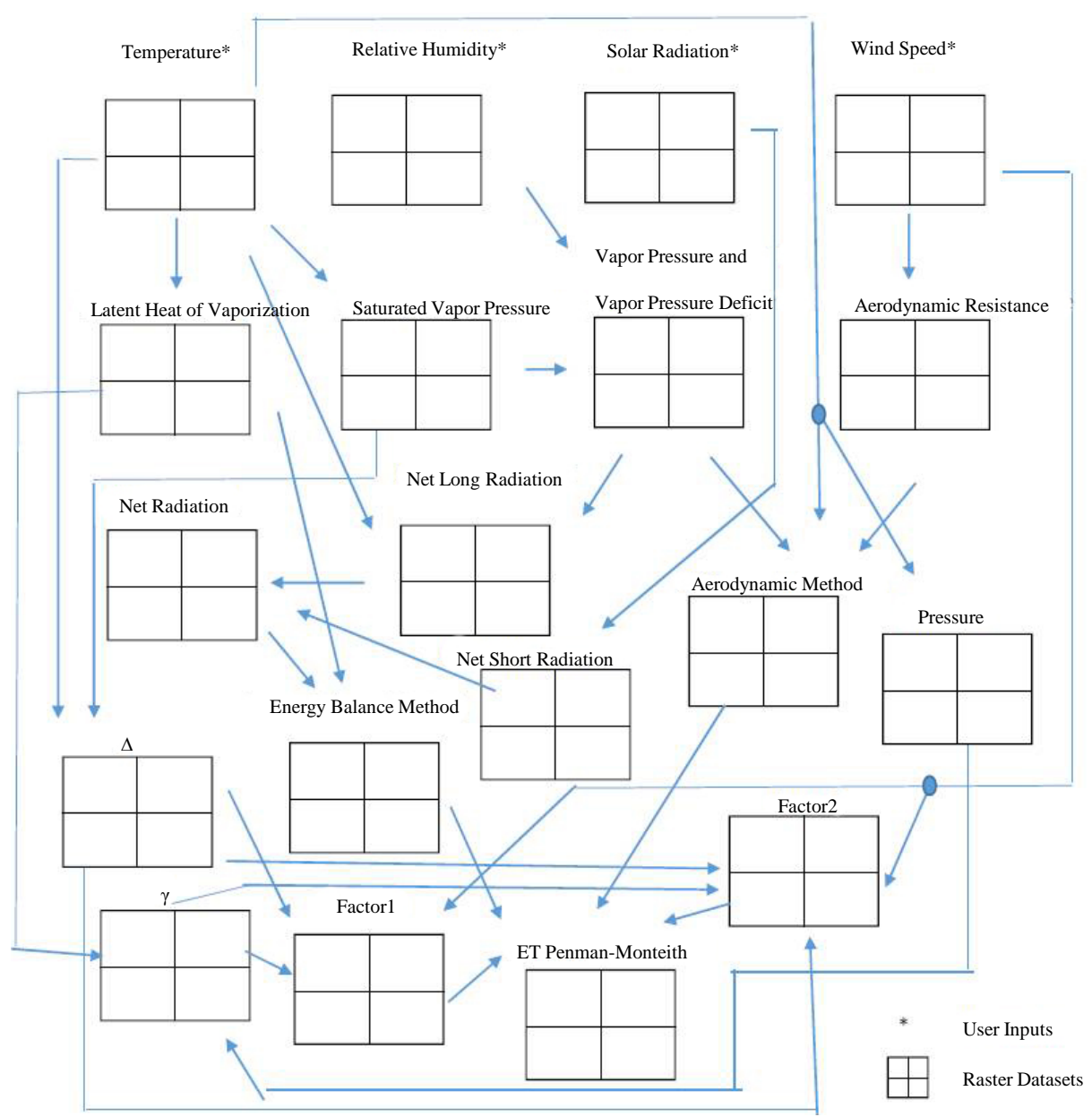

Figure 2. The spatial operations in computing ET using Penman-Monteith.

and the derivative of the function are quite complex. On the other hand, in OAT method which the developed tool uses, at a time, one of the parameters is varied while keeping the other parameters at their base or nominal value. Based on how the parameters are varied with respect to the nominal value, many variations of this method have been proposed. To represent the entire spectrum of the parameter variability, it has been recommended to vary each parameter by certain percentiles while keeping the other parameters at their base value [14]. The percentile variation could be based on the probable minimum and maximum of the parameters, which are based on mean and the standard deviation of the parameters.

\section{Sensitivity Index}

In the developed spatial sensitivity tool, since there many variables associated in the sensitivity analysis, to summarize the outcome of sensitivity analysis using OAT method, a sensitivity index $\left(\Phi_{i}\right)$ is calculated using Equation (18) by varying the parameter from its minimum to its maximum value.

$$
\Phi_{i}=\frac{y_{\max }-y_{\min }}{y_{\max }}
$$

where $y_{\max }$ and $y_{\min }$ are the maximum and the minimum model outcomes, respectively, when the parameter is changed over its entire range (i.e., from its minimum to maximum).

\section{The Development of Spatial Sensitivity Tool at Grid Scale}

ArcGIS provides easy-to-use platform to extend its desktop features by accessing geoprocessing functionalities 
through programming/scripting languages. Python, an open source programming language supported by a growing user community for its extensive collection of standard and third-party libraries, is one of the scripting languages supported by Environmental Systems Research Institute (Esri). The communication between ArcGIS and Python is through a site-package that is called ArcPy. Using the ArcPy site-package, the customization of desktop features could be in three ways: desktop add-in, standard toolbox, Python toolbox. Since the development of spatial sensitivity tool at grid scale does not involve an event such as dragging a rectangle over a geographical map to define an area of interest, Python toolbox is used to develop the spatial sensitivity tool. The developed python toolbox is basically an ASCII-based file, which contains scripts written using Python scripting language and the ArcPy site-package. As shown in Figure 3, the skeleton of ArcGIS Python toolbox is basically a class in Python. A toolbox can have more than one tool. Each tool is defined by a class. The tools are associated with the toolbox class by setting the "tools" property of the toolbox within the constructor or the class initialization method of the toolbox class. As shown in Figure 3, a tool named "SpatialETSense", which is the class for the spatial sensitivity tool, is associated with the toolbox.

The graphical user interface of the developed spatial sensitivity tool is shown in Figure 4. The sample code to retrieve the datasets and the other inputs specified by the user, and the functionalities of the methods (e.g., getParameterInfo () and execute ()) within the "SpatialETSense" class are discussed in detail by [15]. To estimate the spatial ET using Penman-Monteith, this tool adopts the Python source code discussed by [15]. Therefore, only the important chunk of code relevant to sensitivity analysis is discussed in this paper.

Based on the parameter range (i.e., \% of the base value of the parameter at each raster cell) and the number of simulations specified by the user, the interval of sampling is calculated as shown in the variable named "mulRange", where the Python variables "inSenseHigh", "inSenseLow", and "inNumberSimulations" are the maximum value of the parameter, the minimum value of the parameter, and the number of simulations, respectively. The maximum value of the parameter and the minimum value of the parameter are calculated within an if-else block based on the parameter name that is specified by the user and stored by the tool in the Python variable named "inSenseType".

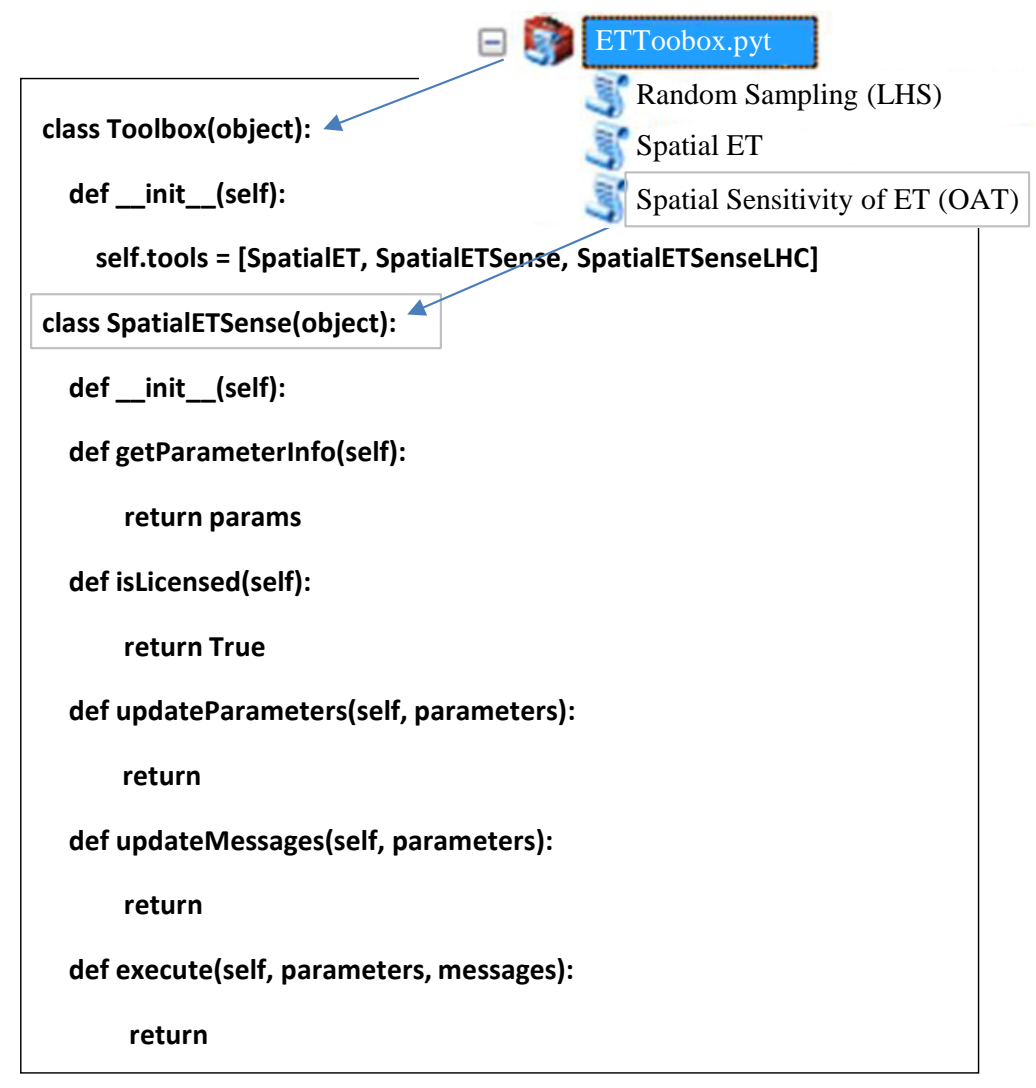

Figure 3. The skeleton of spatial sensitivity tool. 


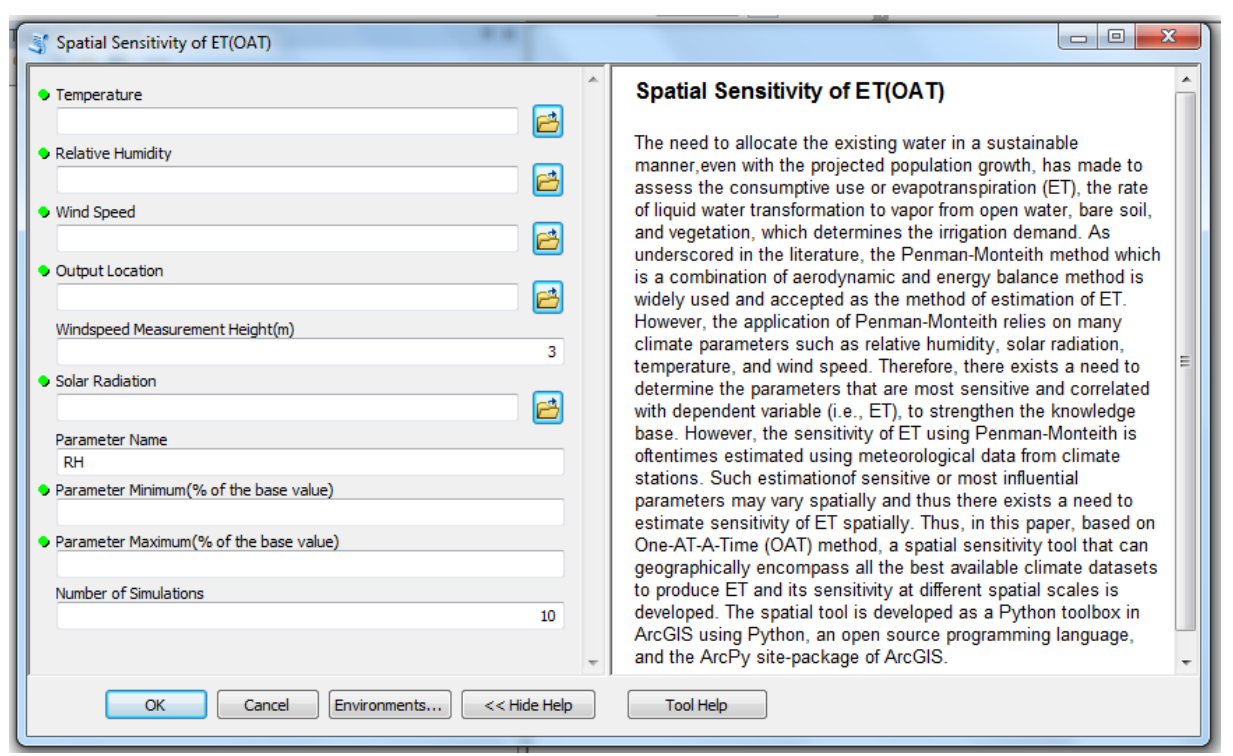

Figure 4. The graphical user interface of spatial sensitivity tool.

if(inSenseType==‘Temp’):

inSenseLow=Raster(inRasterTempF)-parameters[7].value*Raster (inRasterTempF)/100

inSenseHigh=Raster(inRasterTempF)+parameters[8].value*Raster (inRasterTempF)/100

elif inSenseType=='RH':

inSenseLow=Raster(inRasterRH) - parameters[7].value*Raster (inRasterRH)/100

inSenseHigh=Raster (inRasterRH)+parameters[8].value*Raster (inRasterRH)/100

elif inSenseType=='Wind':

inSenseLow=Raster (inRasterW)-parameters[7].value*Raster (inRasterW)/100

inSenseHigh=Raster (inRasterW)+parameters[8].value*Raster (inRasterW)/100

elif inSenseType=='Solar':

inSenseLow=Raster (inSolarRadiation)-parameters[7].value*Raster (inSolarRadiation)/100

inSenseHigh=Raster (inSolarRadiation)+parameters[8].value*Raster (inSolarRadiation)/100

inNumberSimulations=parameters [9].value

mulRange $=($ inSenseHigh-inSenseLow $) /($ inNumberSimulations-1)

The below code shows the variables that are used to store the user specified rasters such as the temperature, relative humidity, and wind speed.

inRasterTempC $=($ Raster(inRasterTempF) -32$) / 1.8$

inRasterRH2=Raster(inRasterRH)

Windspeed=Raster(inRasterW)

inSolarR=Raster(inSolarRadiation)

listRaster=“”

As shown below, based on the number of simulations specified by the user, a for-loop is setup. Within the for-loop, the variables that are used to store the user specified rasters such as the temperature, relative humidity, and wind speed, are updated using the Python variable "mulRange" and the iteration number. Since the sensitivity analysis is based on the concept of One-AT-A-Time, within the for-loop, an if-else condition is developed to identify the raster dataset that needs to be modified. For example, if the user specified parameter name is "RH", the raster dataset associated with relative humidity is modified within the for-loop at each iteration. The other raster datasets (i.e., temperature, wind speed, and solar radiation) are kept at their base values for all the iterations.

for $\mathrm{x}$ in range $(0$, inNumberSimulations):

messages.addWarningMessage ("The iteration number $\{0\}$ is going on.”.format (x))

if(inSenseType==‘Temp’):

inRasterTempC $=($ Raster(inRasterTempF)/Raster(inRasterTempF $) *($ mulRange* $x+$ inSenseLow $)-32) / 1.8$

elif inSenseType=='RH': 
inRasterRH2=Raster(inRasterRH)/Raster (inRasterRH)*(mulRange* $x+$ inSenseLow)

elif inSenseType=='Wind':

Windspeed=Raster(inRasterW)/Raster (inRasterW)*(mulRange* $\mathrm{x}+$ inSenseLow)

elif inSenseType=='Solar':

InSolarR=Raster(inSolarRadiation)/Raster(inSolarRadiation)*(mulRange* $\mathrm{x}+$ inSenseLow)

Having generated the raster dataset for the parameter that is tested in the sensitivity analysis, the ET raster is developed as discussed in Section 2.0. The detailed Python code is outlined by [15]. Since the simulation is carried for a specified number of times, the developed ET raster datasets are stored in a systematic way by appending the simulation number. These ET raster datasets are used to "Mosaic" in ArcGIS to get the sensitivity index for each raster cell. Since the mosaic operation is in need of names of ET raster datasets, which are separated by semicolons, a variable named "listRaster" is used to update the string at each iteration using the concatenation operator in Python programming language.

PenmanMon.save(outRaster1+str (x))

listRaster=listRaster+outRaster1+str(x)+“;”

As shown in the below code, since the sensitivity index discussed in Section 3.1 requires the maximum and the minimum values of ET at each raster cell, two new raster datasets are created to store this information. The copy_management tool of ArcGIS is used to generate the rasters.

arcpy.Copy_management(outRaster1+str(x), outRaster1+“max”) \#Creating a new raster to store the max raster from sense analysis

arcpy.Copy_management(outRaster1+str(x), outRaster1+“min”) \#Creating a new raster to store the min raster from sense analysis

The mosaic operation on ET raster datasets as outlined in Figure 5 was used to generate the maximum and minimum values of ET spatially (i.e., at each raster cell) for the simulations. The mosaic operation requires a string that contains the raster datasets that need to be analyzed to get the maximum/minimum for each raster cells. This string (i.e., the Python variable named "listRaster") was generated as discussed previously. The second argument of the mosaic tool is the raster dataset that holds the maximum or minimum values of ET spatially. These two rasters were also developed as discussed previously. The raster for the sensitivity index was developed based on the equation discussed in Section 3.1. This raster is saved to the location specified by the user through the graphical user interface of the tool.

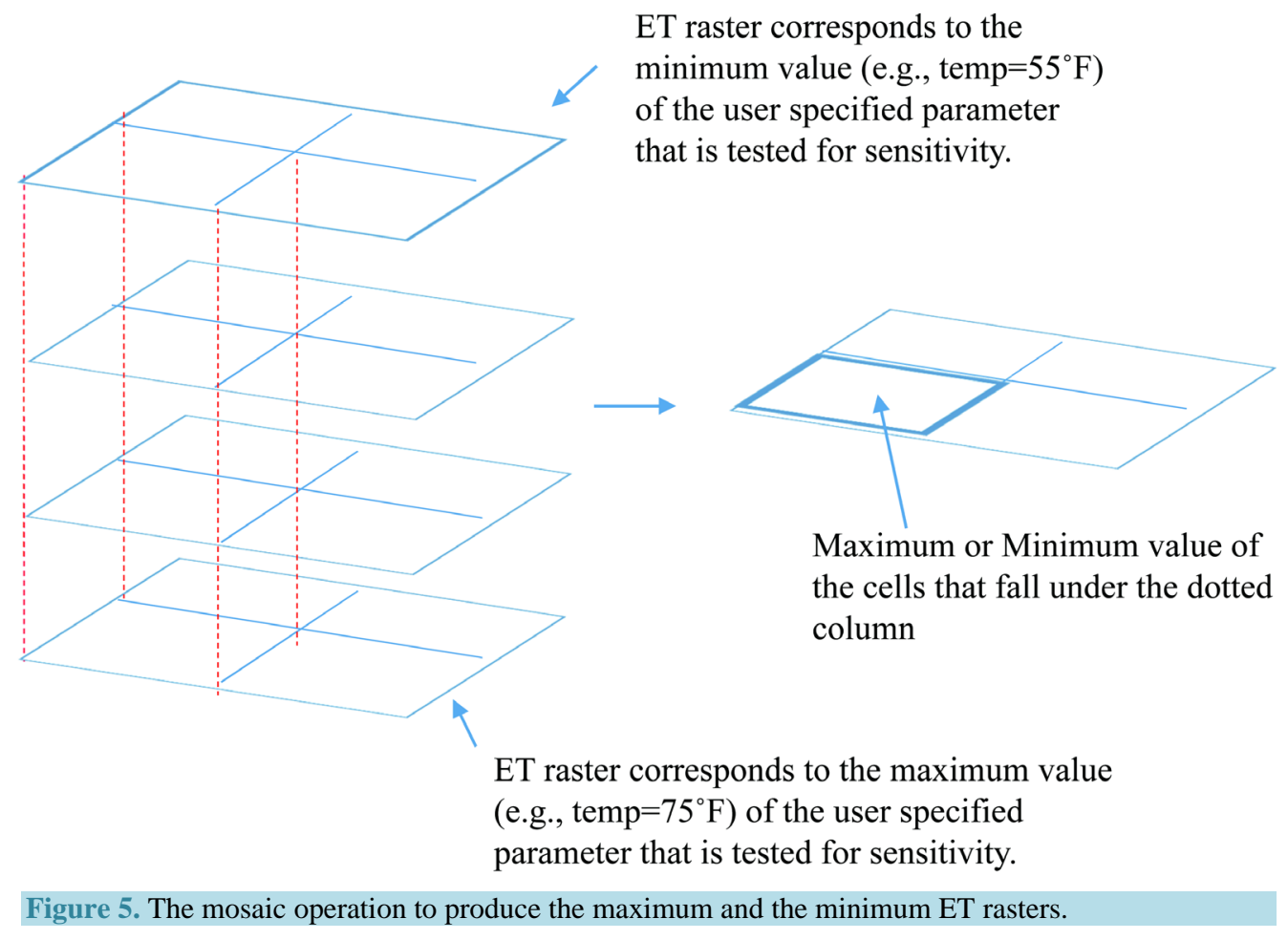


arcpy.Mosaic_management(listRaster,outRaster1+“max”, “MAXIMUM”, “FIRST”, “0”, “”, “””, “”, “””) arcpy.Mosaic_management(listRaster,outRaster1+“min”,"MINIMUM”, “FIRST”, “0”, “”, “””, “””, “””) SenseRaster=(Raster(outRaster1+“max”)-Raster(outRaster1+“min”))/Raster(outRaster1+“max”) SenseRaster.save(outRaster1+“SenseIndex”)

As discussed previously, within each iterations, the ET raster datasets are generated to run the mosaic operation to get the maximum and the minimum ET values for the considered number of simulations for each parameter within the given range. After the mosaic operation, the below lines of code with a for-loop are used to delete the ET raster datasets that were used as inputs for the mosaic operation.

for $\mathrm{x}$ in range $(0$, inNumberSimulations):

arcpy.Delete_management(outRaster1+str (x))

\section{The Application of Spatial Sensitivity Tool}

The state of Nebraska that lies in both the Great Plains and the Midwestern United States has a total geographical area of $200,520 \mathrm{~km}^{2}$. The total population of the state is 1.8 Million [16]. Both the surface and groundwater are used to meet the demand for wide range of purposes. Around $94.8 \%$ of the estimated total groundwater withdrawals is used to meet the irrigation demand [17]. As of November 2014, around 95,000 irrigation wells are registered in the state. The developed tool is demonstrated for the state of Nebraska using the meteorological data from Automated Weather Data Network (AWDN) that gathers climatological observational data and the information for High Plains Region and provides its shareholders in fields such as agriculture. Figure 6 shows the spatial locations of the climate stations in AWDN.

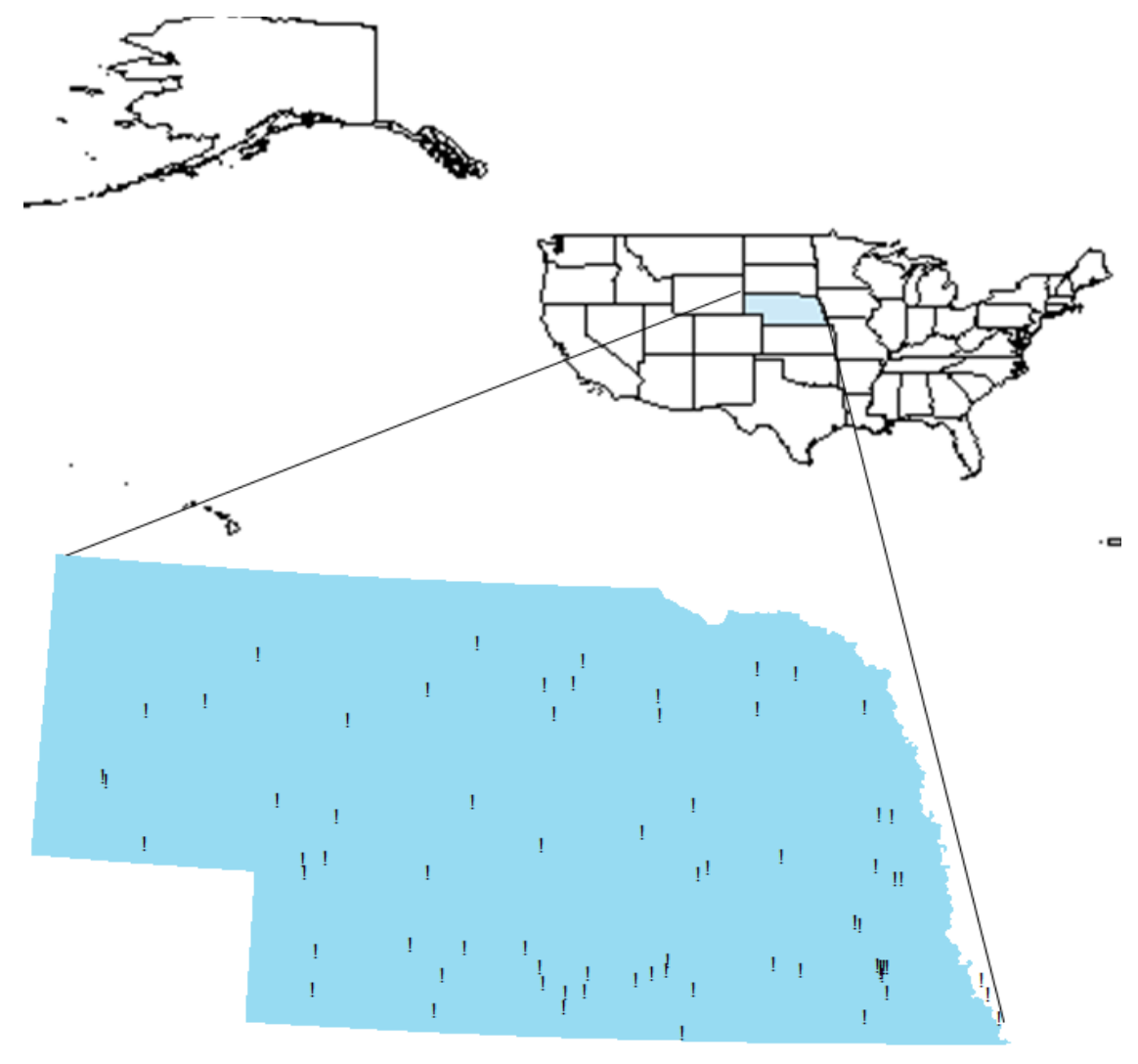

Legend

! AWDN Climate Stations

Nebraska Boundary

Figure 6. The spatial locations of the climate stations from AWDN network in Nebraska. 
The AWDN has around 63 stations to cover the state of Nebraska. The data is available on hourly, daily, and sub-daily basis since 1985 . To demonstrate the tool, the daily data in 2010 was downloaded from the online services provided by AWDN. As discussed in Section 2.0, the developed tool requires raster datasets on temperature, relative humidity, wind speed, and solar radiation. Therefore, at first, the average daily data in 2010 was developed based on the daily data of temperature, relative humidity, and wind speed. Using ArcGIS and the spatial locations of the climate stations, the tabular datasets of average daily data in 2010 were transformed to geographical data. Subsequently, the Spatial Analyst Extension of ArcGIS was used to develop the grid level values of temperature, relative humidity, and wind speed at a resolution of $1 \mathrm{~km}$. The Kriging spatial interpolation technique packaged with ArcGIS was used to develop the rasters of required inputs. To ensure that the Krigged data covers the whole state, the extent of the interpolation was set using the state map of Nebraska. The research work carried out by [4] was used to develop the solar radiation raster.

The estimated ET using Penman-Monteith method is shown in Figure 7(a). Figure 7(b) shows the categorized version of Figure 7(a). For the state of Nebraska in 2010, the estimated ET using Penman-Monteith method varies from 0.77 to $1.04 \mathrm{~mm} /$ day. In other words, the maximum spatial variation of estimated ET using Penman-Monteith method is $=\frac{1.04 \mathrm{~mm} / \text { day }-0.77 \mathrm{~mm} / \text { day }}{1.04 \mathrm{~mm} / \text { day }} \times 100 \%=26 \%$. Moreover, the highest estimated ET using Penman-Monteith method is registered in the Eastern part of Nebraska. However, the geographical extent of such high ET is limited to a very small area in contrast to the geographical area with estimated ET using Penman-Monteith method in the range of 0.77 - 0.96, as shown in Figure 7(b). Furthermore, an increasing trend of spatial variation is observed from Western part of Nebraska to Eastern part of Nebraska in the direction of North West to South East.

Figures 8(a)-(d) show the maps of spatial sensitivity index of ET using Penman-Monteith method for relative humidity, solar radiation, wind, and temperature, respectively. For these maps, as indicated in the legends of the maps, the number of simulations was set to 50 , and the variation of the said parameters were set to $25 \%$ with respect to the base value for each raster cell. In other words, for each raster cell, the maximum and the minimum are considered as $125 \%$ and $75 \%$ of the base value for each raster cell, respectively. As can be observed from Figure 8, among the considered parameters in the sensitivity analysis of ET using Penman-Monteith, solar radiation has the highest sensitivity index in the range of $0.50-0.51$, followed by temperature in the range of 0.40 0.42 for the state of Nebraska. The computed sensitivity index of wind speed and the relative humidity are not that significant compared to the sensitivity index of solar radiation and temperature. The sensitivity index of relative humidity is the lowest among the considered parameters. It is also noted that the spatial sensitivity index of solar radiation increases in the direction of South East to North West, in contrast to the trend of the computed ET that is shown in Figure 7. In other words, the regions that have higher ET and the regions that have higher spatial sensitivity index of solar radiation are not the same. Moreover, it is also observable that the regions that have lower ET (i.e., in the direction of North West) are associated with lower spatial sensitivity index of

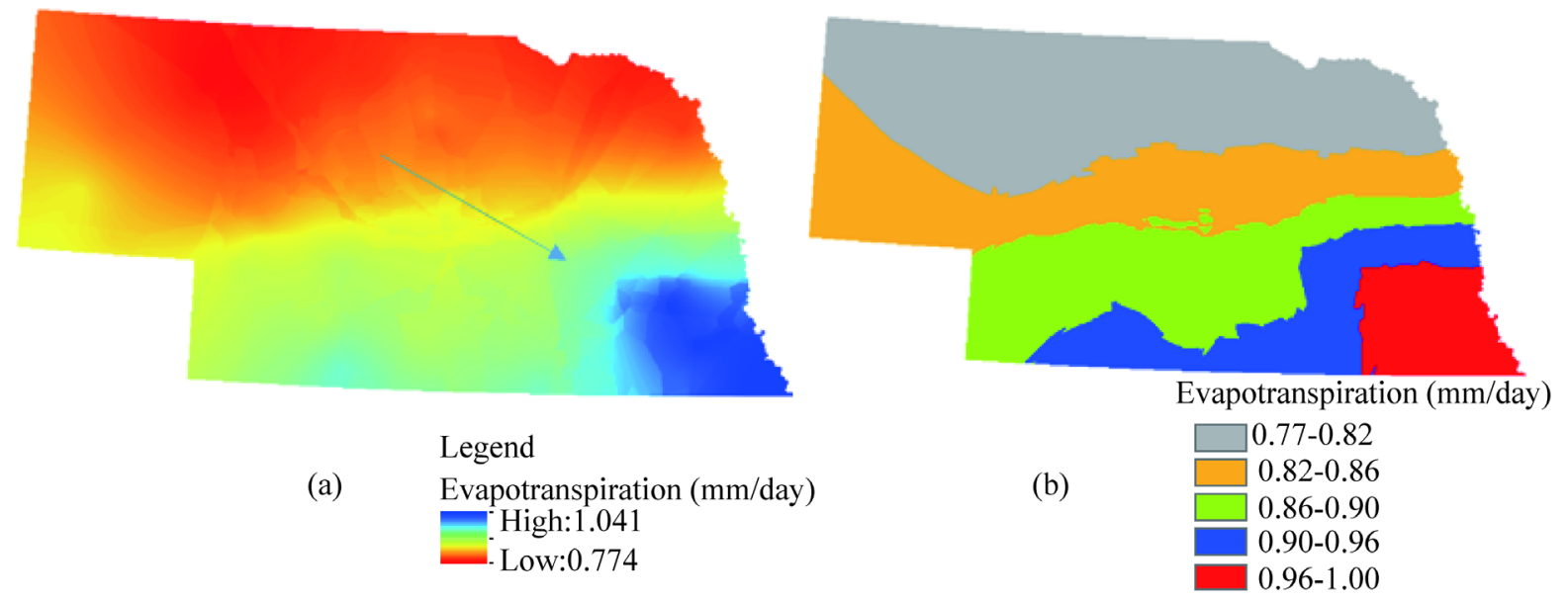

Figure 7. The estimation of ET (mm/day) using Penman-Monteith method. 


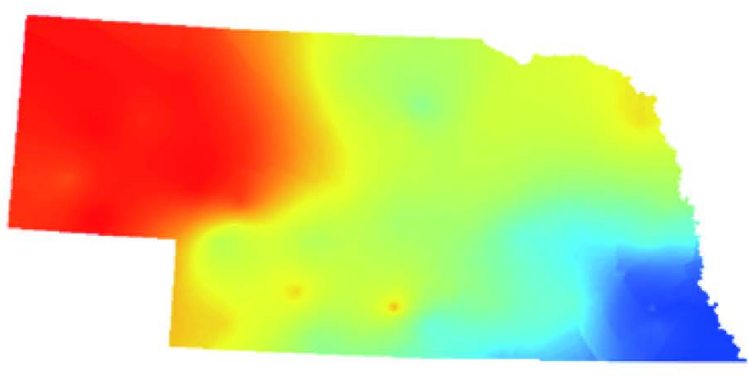

Legend RH_50_25

(a)

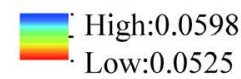

Low:0.0525

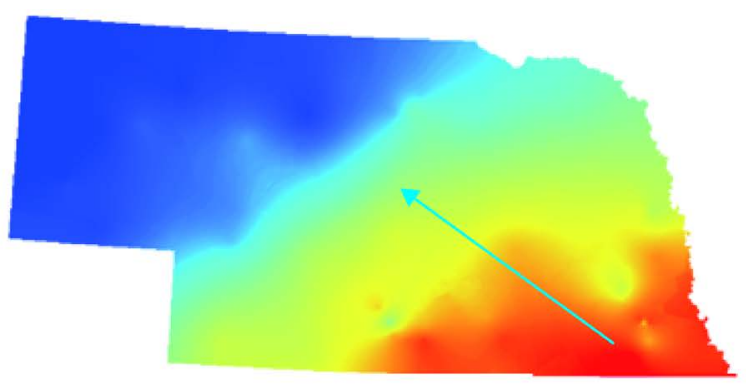

Legend Solar $50 \quad 25$

-High:0.5137

(b)

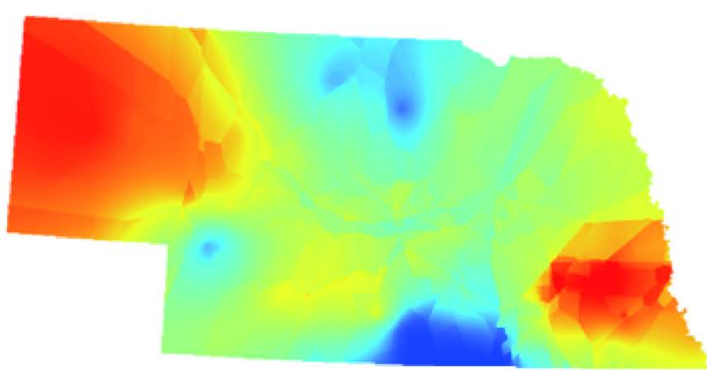

Legend

Temp_50_25

(d)

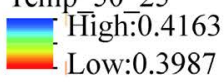

(d)

High:0.1688

Low:0.3987

Figure 8. The spatial sensitivity index of ET using Penman-Monteith method for (a) relative humidity, (b) solar radiation, (c) wind speed, and (d) temperature.

temperature, but higher spatial sensitivity index of solar radiation.

Figures 9(a)-(c) show the spatial sensitivity index of ET using energy balance method for relative humidity, solar radiation, and temperature, respectively. Since the energy balance method discussed in Section 2.2 is not influenced by wind speed, the spatial sensitivity index of ET using energy balance method for wind speed is not shown in Figure 9. As in the case of ET using Penman-Monteith, the solar radiation is the most influencing parameter on the computed ET using energy balance method. However, it is worth to note that although the temperature raster is the second sensitive parameter on ET using Penman-Monteith as pronounced through its magnitude of sensitivity index (i.e., 0.40 - 0.42), the magnitude of sensitivity index(i.e., $0.04-0.06$ ) has diminished in influencing the ET using energy balance method. In other words, the ET using energy balance method is sensitive to solar radiation, whereas the ET using Penman-Monteith is sensitive for both the solar radiation and the temperature. It is also noted that the spatial sensitivity index of solar radiation increases in the direction of South East to North West, similar to what is observed in Figure 7.

Similarly, Figure 10(a) and Figure 10(b) show the variation of spatial sensitivity of ET using aerodynamic method for temperature and relative humidity, respectively. The spatial sensitivity of ET using aerodynamic method for wind speed is not shown as the computed sensitivity index was zero. Since the aerodynamic method discussed in Section 2.1 is not influenced by solar radiation, the spatial sensitivity index of ET using aerodynamic method for solar radiation is not shown in Figure 10. In other words, in contrast to ET using PenmanMonteith and ET using energy balance method, solar radiation is not a significant factor in determining the ET using aerodynamic method. The relative humidity followed by temperature is the most influencing parameter in the computation of ET using aerodynamic method. Moreover, the spatial sensitivity index of ET using aerodynamic 


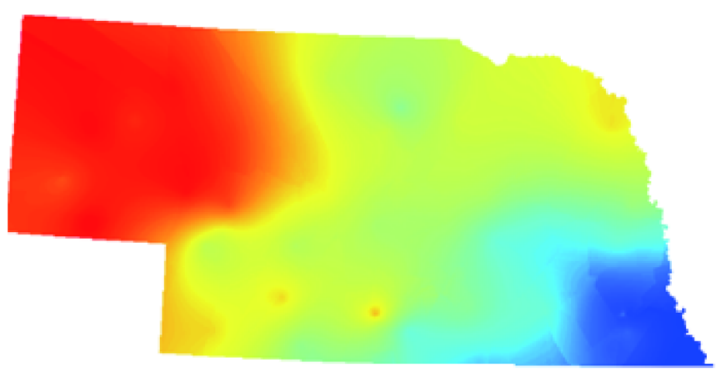

Legend

(a)

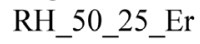

High:0.0598

Low:0.0525

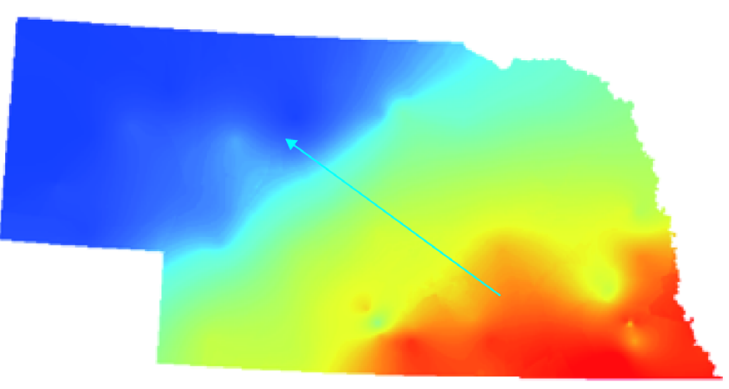

Legend

Solar_50_25_Er

(b)

Low:0.5030

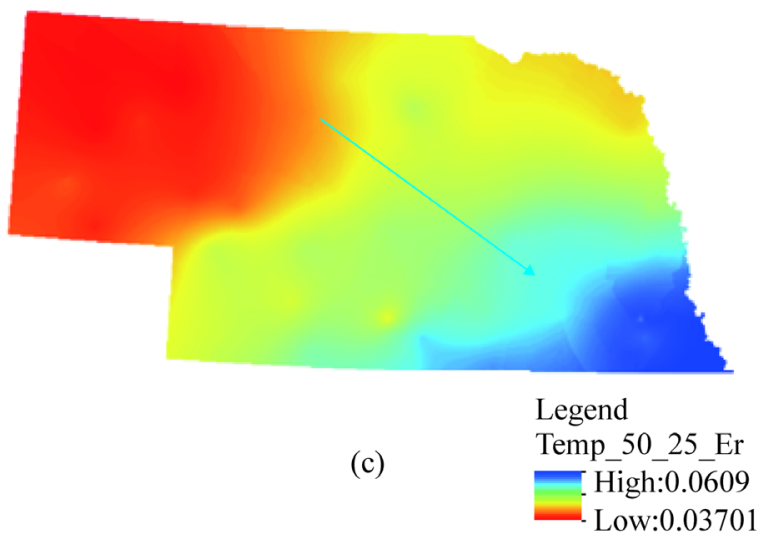

Figure 9. The spatial sensitivity index of ET using energy balance method for (a) relative humidity, (b) solar radiation, and (c) temperature.

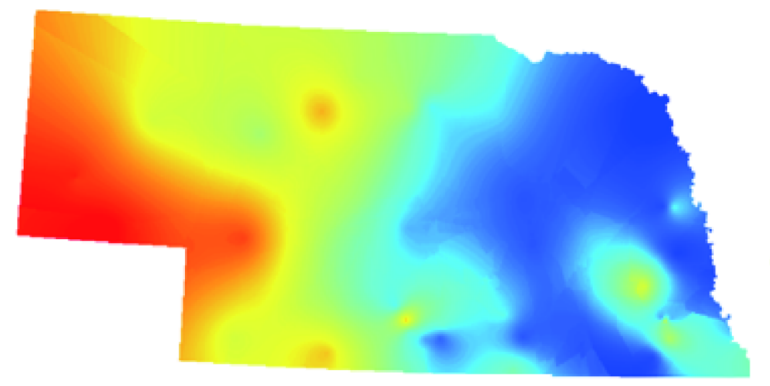

Legend

RH_50_25_Ea

(a)

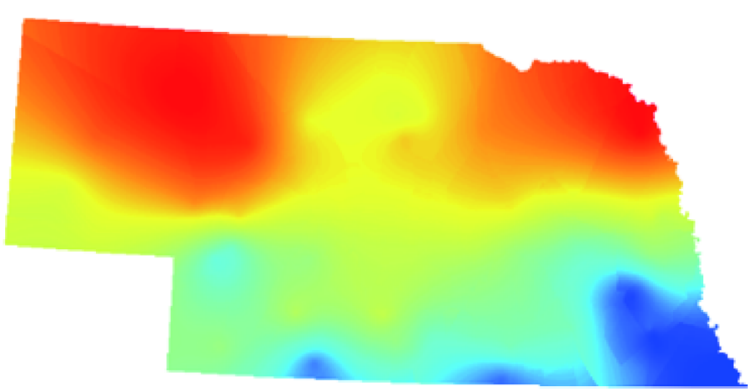

Legend Temp 5025 Ea

(b)

Figure 10. The spatial sensitivity index of ET using aerodynamic method for (a) relative humidity, and (b) temperature.

method is higher than the spatial sensitivity index of ET using Penman-Monteith and the energy balance method.

To understand the influence of parameter variation (as a \% of the base value for each raster cell) on the trend of computed spatial sensitivity index, simulations were carried out for parameter variations of $5 \%, 10 \%, 15 \%$, $20 \%$, and $25 \%$ of the base value for each raster cell. As previously, the number of simulations was fixed to 50 . The outcome of these simulations is placed in Figure 11 and Figure 12. Figure 11 and Figure 12 show the 
maximum and minimum sensitivity index registered in the study area, respectively, for the parameter variations outlined above. Both the maximum and minimum sensitivity index vary linearly and increase as the considered

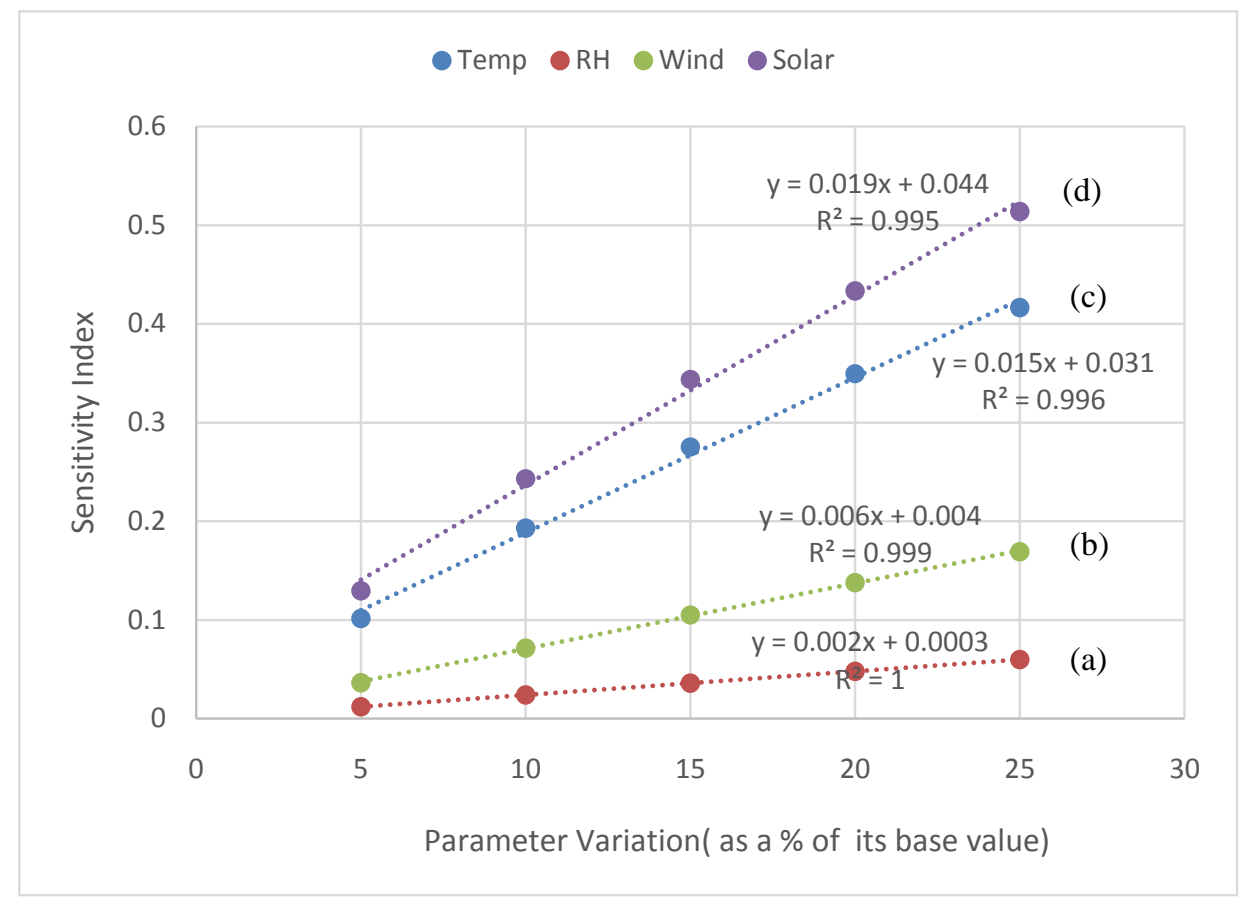

Figure 11. The maximum spatial sensitivity index of ET using Penman-Monteith method for different parameter variation for (a) relative humidity, (b) wind speed, (c) temperature, and (d) solar radiation.

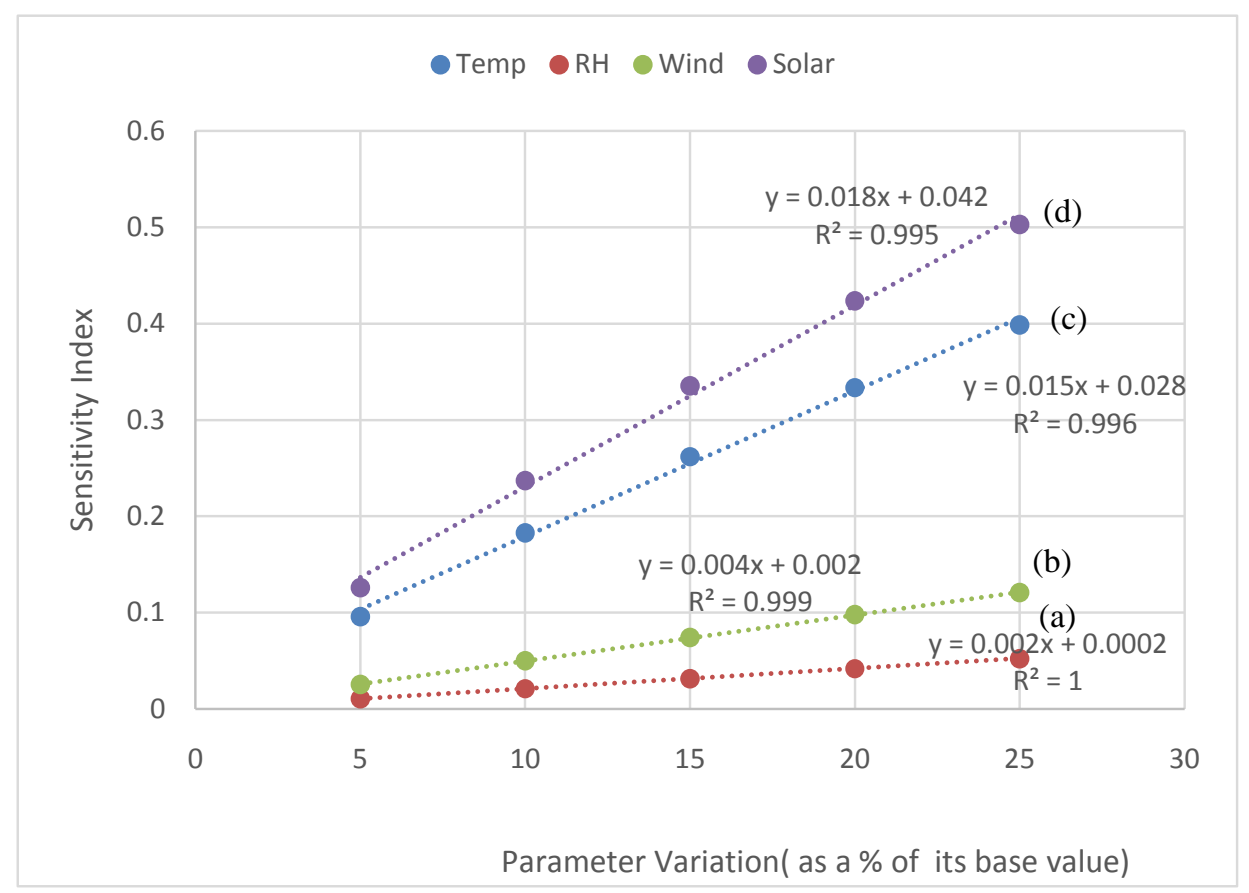

Figure 12. The minimum spatial sensitivity index of ET using Penman-Monteith method for different parameter variation for (a) relative humidity, (b) wind speed, (c) temperature, and (d) solar radiation. 
parameter variations increase. In other words, with more variation expressed with the base value, the computed maximum and minimum sensitivity index also increase for all the parameters. The range that is denoted between the minimum and the maximum value of the computed sensitivity index for the selected parameters, too increases with the increased parameter variations. Furthermore, the fitted equations whose strengths are explained through the statistical measure $\left(\mathrm{R}^{2}\right)$ also reveal that the rate of increase with the increase of parameter variation decreases in the order of solar radiation, temperature, wind speed, and relative humidity.

\section{Conclusion and Recommendations}

In this paper, a spatial sensitivity tool that can geographically encompass all the best available climate datasets to produce ET and its sensitivity at different spatial scales is developed. Based on the outcome of this study, the following points are highlighted:

1) Among the considered parameters in the sensitivity analysis of ET using Penman-Monteith, solar radiation registers the highest sensitivity index followed by the temperature for the state of Nebraska. The computed sensitivity index of wind speed and the relative humidity are not that significant compared to the sensitivity index of solar radiation and temperature.

2) Though the local sensitivity analysis such as One-AT-A-Time is the simplest method of sensitive analysis, the combined variability resulting from changing all the parameters simultaneously is precluded. Therefore, extending the developed tool with random sampling methods such as Latin Hypercube could be one of the future research needs.

3) In demonstrating the developed spatial sensitivity tool, the simulations are carried by varying the base value of the parameters, such as the temperature and solar radiation, for each raster cell by a given percentile. The percentile variation of the base value for each raster cell could be based on historical temporal datasets at each raster cell.

4) The developed spatial sensitivity tool is tested for parameters such as temperature, relative humidity, solar radiation, and wind speed. The develop tool can also further be tuned to test the sensitivity of ET with the intermediate or secondary parameters that are derived from the user specified parameters. The developed tool could also be potentially extended to establish multiple regressed equations to predict ET values at each raster cell based on the sensitivity analysis at each raster cell.

\section{Acknowledgements}

The authors would like to thank the Board of Regents, University of Nebraska-Lincoln, Lincoln, for providing the financial support to conduct this research. This research was conducted when author ${ }^{*}$ was a researcher at University of Nebraska-Lincoln, Lincoln.

\section{References}

[1] Postel, S.L., Daily, G.C. and Ehrlich, P.R. (1996) Human Appropriation of Renewable Fresh Water. Science, 271, 785788. http://dx.doi.org/10.1126/science.271.5250.785

[2] WWAP (World Water Assessment Programme) (2012) United Nations World Water Development Report 4: Managing Water under Uncertainty and Risk. United Nations Educational, Scientific and Cultural Organization, Paris.

[3] WWAP (World Water Assessment Programme) (2015) United Nations World Water Development Report 2015: Water for a Sustainable World. United Nations Educational, Scientific and Cultural Organization, Paris.

[4] Jensen, M.E., Burman, R.D. and Allen, R.G. (1990) Evapotranspiration and Irrigation Water Requirements, Manuals and Reports on Engineering Practice. ASCE. No. 70, New York.

[5] Richard, G.A. and William, O.P. (1986) Rational Use of the FAO Blaney-Criddle Formula. Journal of Irrigation and Drainage Engineering, 112, 139-155. http://dx.doi.org/10.1061/(ASCE)0733-9437(1986)112:2(139)

[6] Allen, R.G., Pereira, L.S., Raes, D. and Smith, M. (1998) Crop Evapotranspiration-Guidelines for Computing Crop Water Requirements. FAO Irrigation and Drainage Paper 56. United Nations Food and Agriculture Organization, Rome.

[7] Hou, L.-G., Zou, S.-B., Xiao, H.-L. and Yang, Y.-G. (2010) Sensitivity of the Reference Evapotranspiration to Key Climatic Variables during the Growing Season in the Ejina Oasis Northwest China. SpringerPlus, 2, S4. http://www.springerplus.com/content/2/S1/S4

[8] Gong, L.B., Xu, C.Y., Chen, D.L., Halldin, S. and Chen, Y.Q.D. (2006) Sensitivity of the Penman-Monteith Reference Evapotranspiration to Key Climatic Variables in the Changjiang (Yangtze River) Basin. Journal of Hydrology, 329, 
620-629. http://dx.doi.org/10.1016/j.jhydrol.2006.03.027

[9] Ambas, V.Th. and Baltas, E. (2012) Sensitivity Analysis of Different Evapotranspiration Methods Using a New Sensitivity Coefficient. Global NEST Journal, 14, 335-343.

[10] Montaldo, N., Toninelli, V., Albertson, J.D., Mancini, M. and Troch, P.A. (2003) The Effect of Background Hydrometeorological Conditions on the Sensitivity of Evapotranspiration to Model Parameters: Analysis with Measurements from an Italian Alpine Catchment. Hydrology and Earth System Sciences, 7, 848-861. http://dx.doi.org/10.5194/hess-7-848-2003

[11] Ayat, R., Mohammad, K., Ayob, B. and Mohsen, D. (2013) Comparison of Various Sensitivity Approaches to Estimate Evapotranspiration to Climatic Variables in Fasa-Synoptic Station_Iran. International journal of Advanced Biological and Biomedical Research, 1, 459-466.

[12] Bakhtiari, B. and Liaghat, A.M. (2011) Seasonal Sensitivity Analysis for Climatic Variables of ASCE PenmanMonteith Model in a Semi-Arid Climate. Journal of Agricultural Science and Technology, 13, 1135-1145.

[13] Zha, T., Li, C., Kellomaki, S., Peltola, H. and Wang, K.-Y. (2013) Controls of Evapotranspiration and $\mathrm{CO}_{2}$ Fluxes from Scots Pine by Surface Conductance and Abiotic Factors. PLOS ONE, 8, e69027. http://dx.doi.org/10.1371/journal.pone.0069027

[14] Hamby, D.M. (1994) A Review of Techniques for Parameter Sensitivity Analysis of Environmental Models. Environmental Monitoring and Assessment, 32, 135-154. http://dx.doi.org/10.1007/BF00547132

[15] Mylevaganam, S. and Ray, C. (2016) A Spatial Evapotranspiration Tool at Grid Scale. Open Journal of Applied Sciences, 6, 64-77. http://dx.doi.org/10.4236/ojapps.2016.61007

[16] Annual Estimates of the Resident Population for the United States, Regions, States, and Puerto Rico: April 1, 2010 to July 1, 2014. U.S. Census Bureau. January 7, 2015.

[17] Nebraska Surface Water and Groundwater Use-2005. Nebraska Department of Natural Resources. http://www.dnr.ne.gov/nebraska-surface-water-and-groundwater-use-2005 